\title{
Response of the gut microbiota during the Clostridioides difficile infection in tree shrews mimics those in humans
}

Wenpeng $\mathrm{Gu}^{1,2}$, Wenge $\mathrm{Li}^{3}$, Wenguang Wang ${ }^{1}$, Dexuan Kuang ${ }^{1}$, Wenzhu Zhang ${ }^{3}$, Caixia Lu' ${ }^{1}, \mathrm{Na} \mathrm{Li}^{1}$, Pinfen Tong ${ }^{1}$, Yuanyuan $\mathrm{Han}^{1}$, Xiaomei Sun ${ }^{1}$, Jinxing $\mathrm{Lu}^{3}$, Yuan $\mathrm{Wu}^{3^{*}}$ (D) and Jiejie Dai ${ }^{*^{*}}$

\begin{abstract}
Background: Clostridioides difficile is a major cause of antibiotic associated diarrhea. Several animal models are used to study C. difficile infection (CDI). The tree shrew has recently been developed as a model of primate processes. $C$. difficile infection has not been examined in tree shrews. We infected tree shrews with hyper-virulent $C$. difficile strains and examined the alterations in gut microbiota using 16S rRNA gene sequencing.

Results: $C$. difficile colonized the gastrointestinal tract of tree shrew and caused diarrhea and weight loss. Histopathologic examination indicated structures and mucosal cell destruction in ileal and colonic tissues. The gut microbial community was highly diversity before infection and was dominated by Firmicutes, Fusobacteria, Bacteroidetes, and Proteobacteria. Antibiotic administration decreased the diversity of the gut microbiota and led to an outgrowth of Lactobacillus. The relative abundance of Proteobacteria, Gammaproteobacteria, Enterobacteriales, Lachnospiraceae, Enterobacteriaceae, Escherichia, Blautia, and Tyzzerella increased following C. difficile infection. These taxa could be biomarkers for C. difficile colonization.
\end{abstract}

Conclusions: In general, the disease symptoms, histopathology, and gut microbiota changes following C. difficile infection in tree shrews were similar to those observed in humans.

Keywords: Clostridioides difficile, Tree shrew, Gut microbiota

\section{Background}

Clostridioides difficile is a major cause of antibiotic associated diarrhea in patients after hospitalization and antibiotic administration [1]. C. difficile infection (CDI) caused by toxigenic isolates can result in a wide range of

\footnotetext{
* Correspondence: wuyuan@icdc.cn; djj@imbcams.com.cn

${ }^{3}$ State Key Laboratory of Infectious Disease Prevention and Control, Collaborative Innovation Center for Diagnosis and Treatment of Infectious Diseases, National Institute for Communicable Disease Control and Prevention, Chinese Center for Disease Control and Prevention, Chang bai Road 155, Chang ping District, Beijing 102206, China

${ }^{1}$ Center of Tree Shrew Germplasm Resources, Institute of Medical Biology, Chinese Academy of Medical Sciences and Peking Union Medical College, Yunnan Key Laboratory of Vaccine Research and Development on Severe Infectious Diseases, Yunnan Innovation Team of Standardization and

Application Research in Tree Shrew, Zhao zong Road 66, Kunming 650118, China

Full list of author information is available at the end of the article
}

outcomes, including asymptomatic colonization, diarrhea, life threatening pseudomembranous colitis, or intestinal obstruction [2]. CDI is the most common cause of healthcare-associated diarrhea and colitis, and is responsible for significant morbidity and substantial healthcare costs worldwide $[3,4]$. The primary pathogenic mechanism of $C$. difficile is the production of enterotoxin A (TcdA) and cytotoxin B (TcdB); some $C$. difficile strains can produce a binary toxin, encoded by the $c d t A$ and $c d t B$ genes, that increases pathogenesis [5]. Since emerging in North American in 2005, hyper-virulent $C$. difficile isolates (PCR ribotype RT027) have caused several infections and outbreaks in the United States of America, Canada, and most European countries [6]. Recently, C. difficile RT078 has been reported from both hospitalized patients and

(c) The Author(s). 2020 Open Access This article is licensed under a Creative Commons Attribution 4.0 International License, which permits use, sharing, adaptation, distribution and reproduction in any medium or format, as long as you give appropriate credit to the original author(s) and the source, provide a link to the Creative Commons licence, and indicate if changes were made. The images or other third party material in this article are included in the article's Creative Commons licence, unless indicated otherwise in a credit line to the material. If material is not included in the article's Creative Commons licence and your intended use is not permitted by statutory regulation or exceeds the permitted use, you will need to obtain permission directly from the copyright holder. To view a copy of this licence, visit http://creativecommons.org/licenses/by/4.0/ The Creative Commons Public Domain Dedication waiver (http://creativecommons.org/publicdomain/zero/1.0/) applies to the data made available in this article, unless otherwise stated in a credit line to the data. 
environmental surfaces in eastern China [7]. This emergent strain also causes severe disease, especially in elderly patients [8].

Several animal models have been used to study different aspects of CDI, including pathophysiology, colonization, transmission, recurrence, and the impact of strain variability [9]. Small animals, such as mice, hamsters, and rats, as well as larger animals, such as foals, gnotobiotic piglets, and rhesus monkeys have been used to study CDI [10]. Each laboratory animal has its own application limitations and does not fully reflect the entire pathophysiological process of $C$. difficile infection in humans. For example, primates were rarely used due to their ethical restrictions; the lesion sites of rodents infected by $C$. difficile were different from human patients; germfree animals didn't really show the ecological niche of human intestine. The utility of the animal model mainly depends on the similarities in pathological characteristics with humans. The tree shrew (Tupaia belangeri) belongs to the family Tupaiidae and order Scandentia, and is widely distributed in South and Southeast Asia [11]. Compared with rodents, tree shrews were considered as an alternative animal model for nonhuman primates. Simultaneously, use of the tree shrew as a laboratory animal has increased in recent years due to its low cost of maintenance, life span, small body size, short reproductive cycle, and close relationship to primates [12]. Several human diseases models have been constructed by using this animal [13]. In view of advantages of tree shrews mentioned above, we attempted to use this animal as a novel model for CDI, compared the characteristics of infection with other animals, and explored its feasibility and applicability.

In addition, disruption of the gut microbiota is another important component of CDI [14]. A number of animal models have been developed to examine the relationship between the gut microbial community and $C$. difficile infection $[15,16]$. However existing models do not fully capture the relationship between the human gut microbiota and $C$. difficile pathogenesis. Therefore, we further analyzed the gut microbiota changes of tree shrews during CDI process by using 16SrRNA gene sequencing in this study. The features of intestinal microbial communities among different animals and humans were compared, and the usability of tree shrews as CDI model was further estimated.

\section{Results}

\section{Clinical features}

Nine of 15 infected animals showed clinical signs of CDI, including diarrhea and weight loss (Fig. 1). The remaining animals did not exhibit signs of diseases despite being colonized with $C$. difficile. Four days after infection, the animals began suffering diarrhea (i.e., loose stools containing mucous) (Fig. 1.A to D). There was not a statistically significant difference in weight loss between groups (each strain represented a group) (Fig. 1.E, $\mathrm{F}=0.115, P=0.892$; average weights $13.35 \pm 2.79 \mathrm{~g}$ for CD21062, $12.68 \pm 1.71 \mathrm{~g}$ for CD10010, and $12.92 \pm 2.13 \mathrm{~g}$ for CD12038). No animals died during the experiment. Fecal samples were all positive for $t c d A, t c d B, c d t A$, and $c d t B$ two days after infection. Fecal samples were toxin negative before infection. The control group for gross anatomy of the abdominal cavity showed normal gut structure of the tree shrew, without any congestion, bleeding or inflammation (Additional file 1.A). However, the infected group of animal indicated the typical inflammation for anatomy of the abdominal cavity, the mesenterium of the tree shrew emerged bled or congestion, and the gut structure disorders could be found (Additional file 1.B).

\section{Elisa}

TcdA toxin could be detected at day 4 after oral gavage of the animals, shown as Fig. 2. A-C. CD10010 and CD12038 strain produced TcdA toxin from two tree shrews at day 4, while toxin could be detected at day 5 for CD21062 strain. At experimental day 5, most of the animal feces could be identify the TcdA. Similar results of TcdB toxin were showed from Fig. 2. D-F. At day 5, both TcdA and TcdB reached the higher levels for some of the tree shrews, after that, the concentration of toxins were relatively stable.

\section{Histopathology}

Histopathology changes were detected in the ileal and colonic tissues of all animals. In healthy ileal and colonic tissues, mucosal cells and structures were arranged neatly and lacked congestion, edema, and inflammatory infiltration (Figs. 3.A to D). Pathology following infection showed evidence of epithelial cell destruction, structural disorders, mucosal ulcers, and necrosis in the mucosa and submucosa of the ileum. Gland and crypt destruction, as well as goblet cell reduction and inflammatory cell infiltration, was observed in mucosal and submucosal layers (Figs. 3.E and F). There was an increase in eosinophil cells, as well as an increase in the infiltration of lymphocyte and plasma cells into colonic tissues in the mucosal and submucosal layers (Figs. 3. G and H).

\section{Taxonomy of the gut microbiota}

3,794,301 reads were obtained from 45 fecal samples. 3, 690,628 reads survived merging and quality trimming. The average read length was $423.62 \pm 6.93 \mathrm{bp}$ and ranged from 408 to $430 \mathrm{bp}$. Reads were mapped to 3675 OTUs.

At baseline (TSCDB group), Firmicutes (64.98\%), Fusobacteria (11.06\%), Bacteroidetes (9.28\%), Proteobacteria (7.79\%), and Epsilonbacteraeota (4.29\%) were the most abundant phyla (Fig. 4.A). Clostridia (40.40\%), 


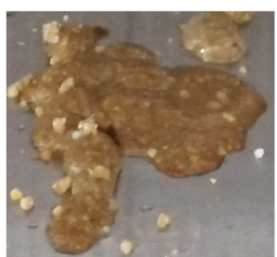

A

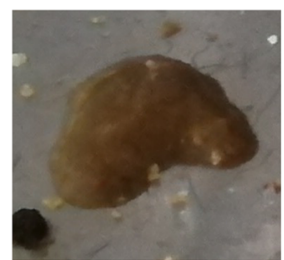

B

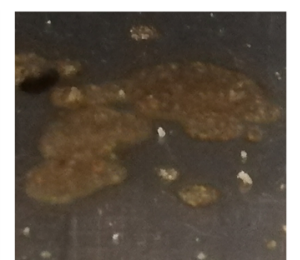

C

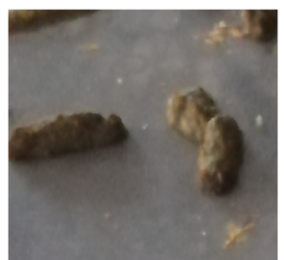

D

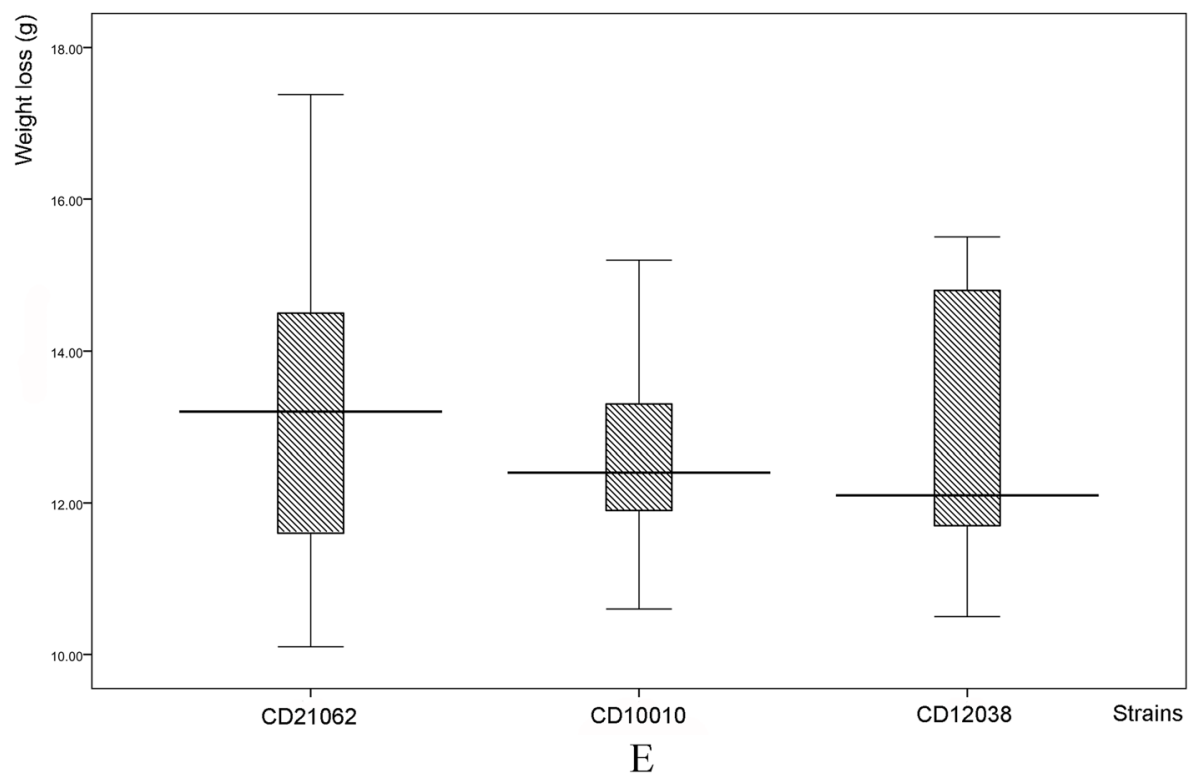

Fig. 1 The disease signs of CDI and weight loss of animal in this study. A to C. loose and mucous stools after tree shrew infected with C. difficile; D. normal control; E. the weight loss of animals in this study

Bacilli (17.28\%), Fusobacteriia (11.06\%), Bacteroidia (9.28\%), and Gammaproteobacteria (6.64\%) were the most abundant classes (Fig. 4.B); Clostridiales (40.40\%), Lactobacillales (14.93\%), Fusobacteriales (11.06\%), Bacteroidales (9.23\%), and Erysipelotrichales (5.13\%) were the most abundant orders (Fig. 4.C). Lachnospiraceae (15.99\%), Peptostreptococcaceae (14.97\%), Streptococcaceae (12.19\%), Fusobacteriaceae (11.06\%), and Bacteroidaceae (5.92\%) (Fig. 4.D) were the most abundant families. Peptoclostridium (13.73\%), Streptococcus (12.19\%), Fusobacterium (11.06\%), Bacteroides (5.92\%), and Clostridium (4.89\%) were the most abundant genera (Fig. 4.E). Streptococcus_porcorum (7.47\%), Trichuris_ trichiura (3.57\%), Staphylococcus_pasteuri (2.24\%), uncultured_Firmicutes_bacterium (1.93\%), and Actinobacillus_indolicus (1.84\%) were the most abundant species (Fig. 4.F).

Antibiotic treatment (TSCDA group) generally decreased the relative abundances of most taxa and caused an outgrowth of Lactobacillus. Firmicutes (96.87\%), Bacilli (96.12\%), Lactobacillales (95.80\%), Lactobacillaceae (94.46\%), and Lactobacillus (94.46\%) were the dominant phyla, class, order, family, and genera, respectively (Fig. 4).
The relative abundances of some taxa recovered followed by $C$. difficile infection (TSCDI group), such as Proteobacteria (phylum, 14.28\%), Clostridia (class, 17.11\%), Gammaproteobacteria (class, 14.18\%); Clostridiales (order, 17.11\%), Enterobacteriales (order, 13.92\%); Enterobacteriaceae (family, 13.92\%), Lachnospiraceae (family, 8.45\%) and Clostridiaceae (family, 7.94\%), Escherichia (genus, 13.14\%), Clostridium (genus, 7.94\%), Roseburia (genus, 2.47\%), and Tyzzerella (genus, 1.95\%) (Fig. 4). The communities during the antibiotic and infection phase were more similar to each other than to the baseline community (Fig. 4).

\section{Diversity analysis}

There were statistically significant differences in alpha diversity (Richness, Shannon index, ACE index, and Simpson's index all $P<0.05)$ between TSCDB, TSCDA and TSCDI groups (Table 1). A PCoA plot based on the UniFrac distance matrix revealed clustering by group (Fig. 5.A) and statistically significant differences between groups (Anosim $\mathrm{R}=0.602, P=0.001$; Fig. 5.B).

A cladogram based on LEfSe analysis showed the phylogenetic distribution of microbial communities in different groups (Fig. 5.C). The dominant taxa at baseline 


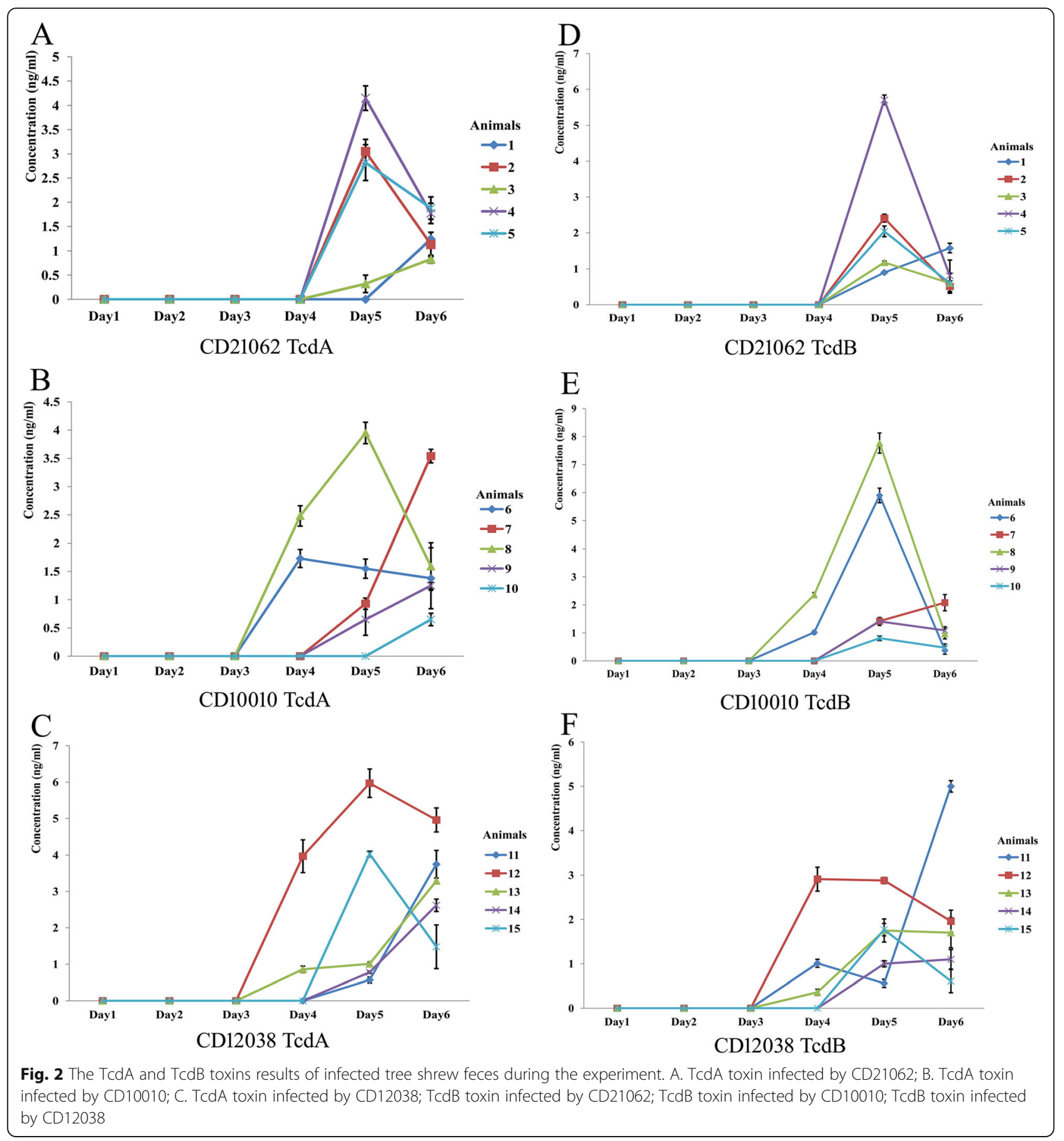

were Firmicutes (Clostridia, Clostridiales and Erysipelotrichia, Erysipelotrichales), Bacteroidetes (Bacteroidia, Bacteroidales, and Bacteroidaceae), Fusobacteria (Fusobacteriia, Fusobacteriales, and Fusobacteriaceae) and Epsilonbacteraeota (Campylobacteria and Campylobacterales). The dominant taxa during antibiotic treatment was Bacilli (Lactobacillales, Lactobacillaceae, and Lactobacillus). After C. difficile infection, the relative abundance of Clostridiales (Clostridiaceae and Clostridium), Ruminococcus, and
Tyzzerella increased. The relative abundance of most taxa decreased between antibiotic treatment and infection (Wilcoxon test). In addition to Clostridium, the relative abundance of Proteobacteria, Gammaproteobacteria, Enterobacteriales, Lachnospiraceae, Enterobacteriaceae, Escherichia, Blautia, and Tyzzerella increased (Table 2). COG and KEGG pathway annotation results based on PICRUSt revealed an increase in the abundance of energy production and conversion; amino acid transport 


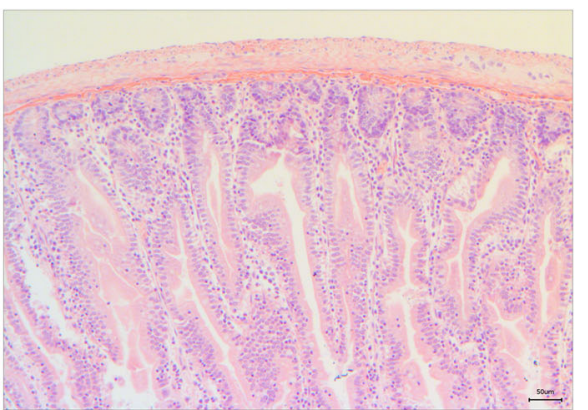

A. Normal ileum $(100 \times)$

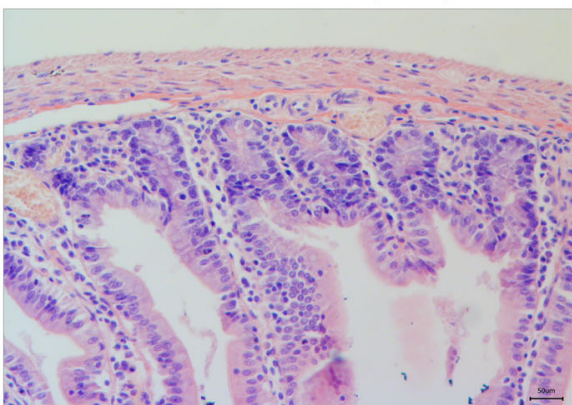

B. Normal ileum $(200 \times)$

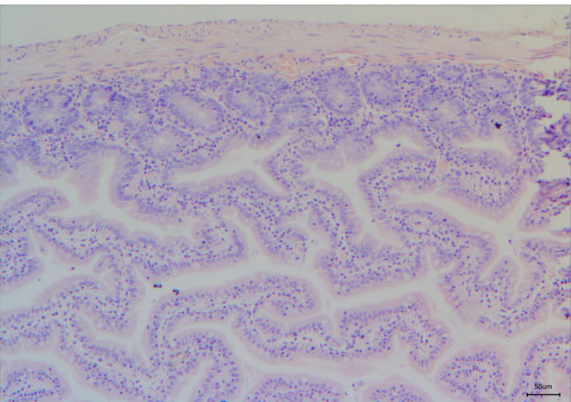

C. Normal colon $(100 \times)$

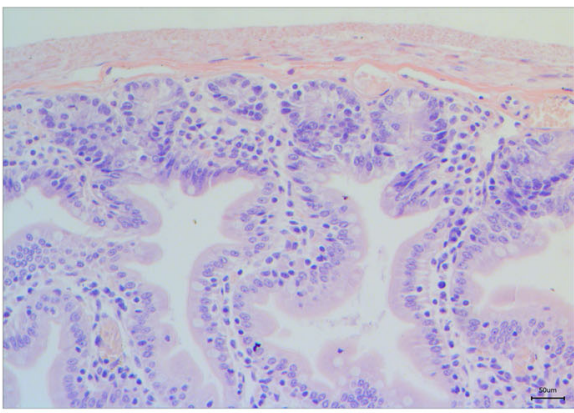

D. Normal colon $(200 \times)$

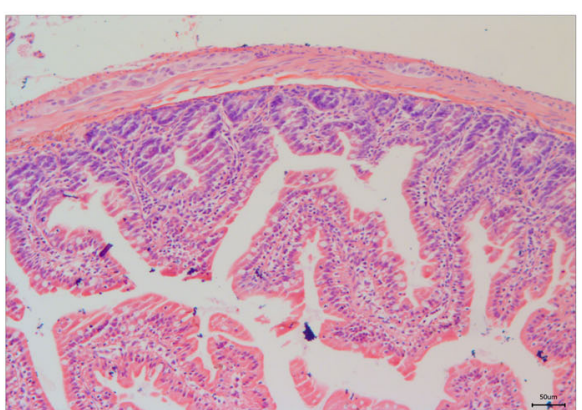

E. Infected ileum $(100 \times)$

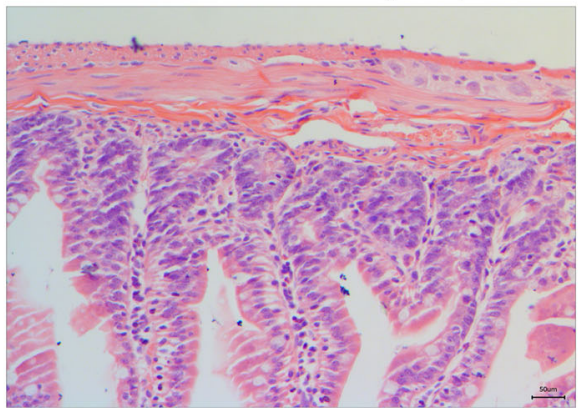

F. Infected ileum $(200 \times)$

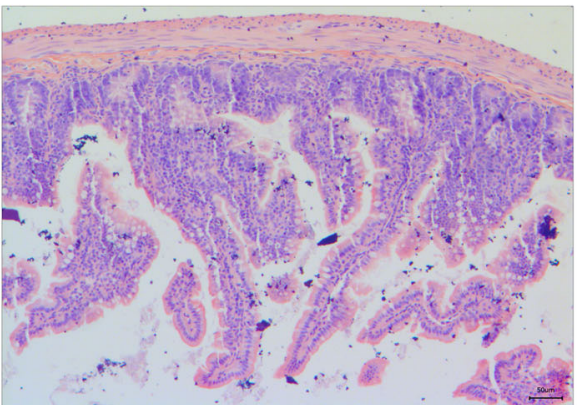

G. Infected colon $(100 \times)$

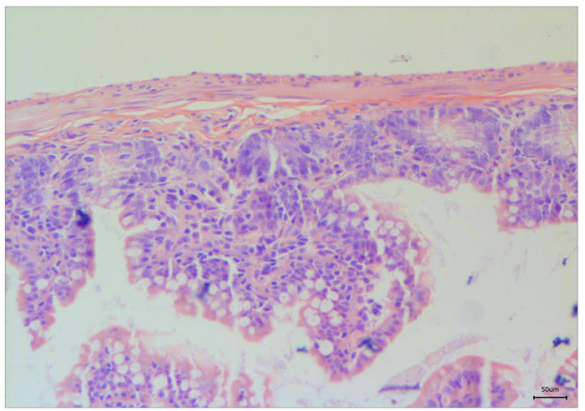

H. Infected colon $(200 \times)$

Fig. 3 Histopathologic examination of ileum and colon of tree shrew infected with C. difficile. A. Normal ileum (100x); B. Normal ileum (200x); C. Normal colon (100x); D. Normal colon (200x); E. infected ileum (100X); F. infected ileum (200X); G. infected colon (100X); H. infected colon (200X)

and metabolism; carbohydrate transport and metabolism; transcription; replication, recombination and repair; cell wall/membrane/envelop biogenesis; signal transduction mechanisms; defense mechanisms in COG pathways, and amino acid metabolism; carbohydrate metabolism; cell motility; energy metabolism; infectious diseases; replication and repair; transcription; translation; cellular processes and signaling in KEGG pathways after $C$. difficile infection (TSCDI group) compared to antibiotic treatment (TSCDA group). Functional predictions revealed that bacterial reproduction and metabolism increased after $C$. difficile infection (Additional file 2). 


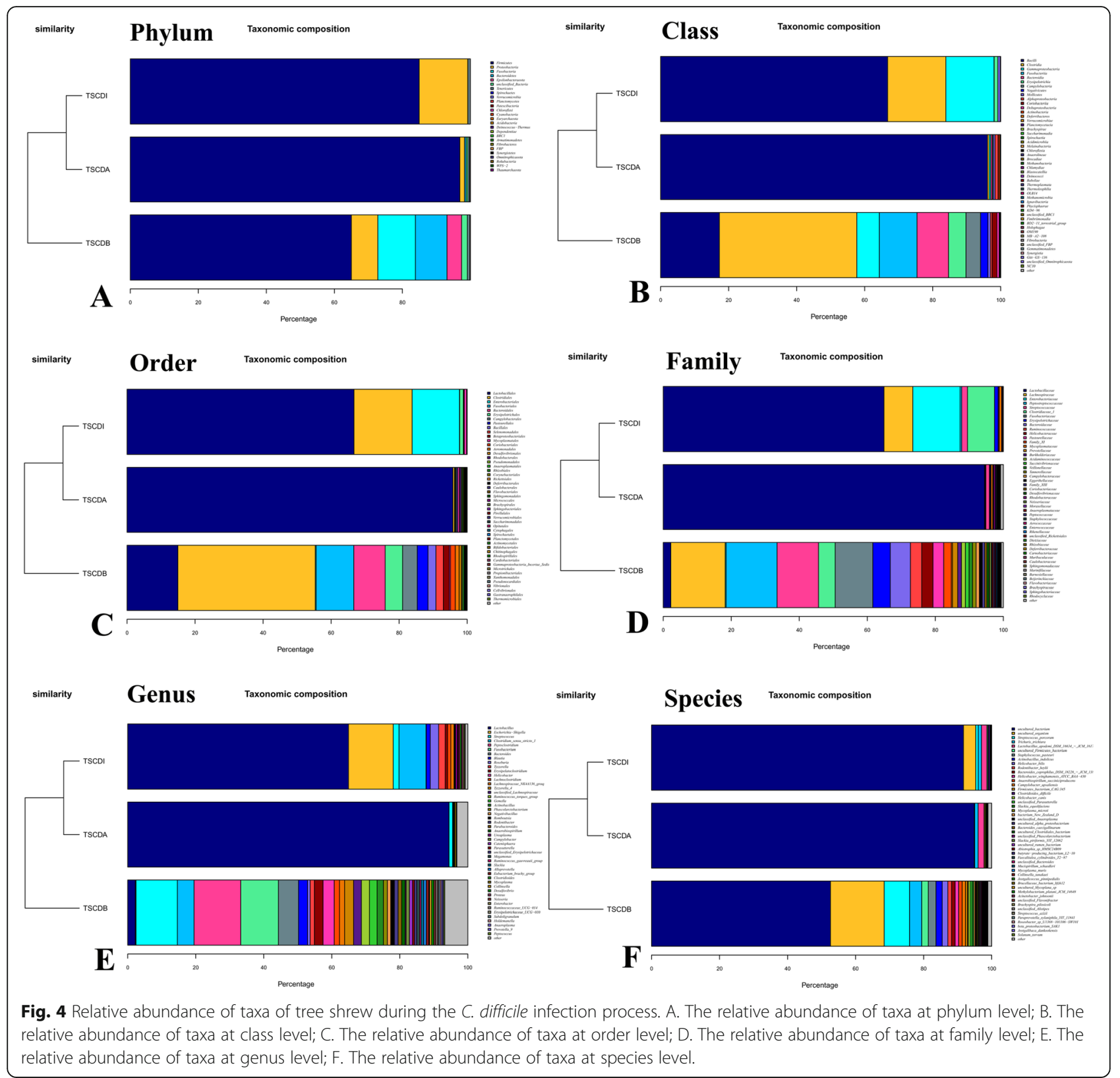

However, the abundance of COG and KEGG pathways were highest at baseline in TSCDB group compared with TSCDA and TSCDI group.

\section{Discussion}

Animal models of human diseases are generally used in the early phases of the understanding of the pathophysiology of a disease, especially when human studies are not feasible for ethical concerns or practical reasons. Although studies on animal models of $C$. difficile infection have been reported, there were still some problems. Thus, the animal model research of $C$. difficile was still necessary. Animal models with high similarities to humans are desirable for studying the pathophysiology and mechanisms of disease [13]. Use of large primates is generally prohibited for ethical reasons. Rodents are commonly used, but these models do not always reflect human pathology [17-19]. Tree shrews are a good model for human biochemical metabolism, physiological function, and genomics [12], and have been widely employed in recent decades. However, this study was the first to investigate $C$. difficile infection in tree shrews. Animals developed diarrhea and weight loss, and histopathology revealed an inflammatory response and 
Table 1 The alpha diversity estimation results in this study

\begin{tabular}{|c|c|c|c|c|c|c|}
\hline Indexs & Groups & Values (mean \pm STD) & $\min$ & $\max$ & Kruskal Wallis H-test & $P$ values \\
\hline \multirow[t]{3}{*}{ Richness } & TSCDB & $430.07 \pm 62.41$ & 291 & 535 & 23.95 & 0.000 \\
\hline & TSCDA & $312.40 \pm 200.43$ & 139 & 847 & & \\
\hline & TSCDI & $188.40 \pm 52.42$ & 111 & 326 & & \\
\hline \multirow[t]{3}{*}{ Shannon entropy } & TSCDB & $3.24 \pm 0.66$ & 2.02 & 4.15 & 31.63 & 0.000 \\
\hline & TSCDA & $0.57 \pm 0.81$ & 0.04 & 2.5 & & \\
\hline & TSCDI & $1.11 \pm 0.61$ & 0.17 & 2.18 & & \\
\hline \multirow[t]{3}{*}{ ACE index } & TSCDB & $851.32 \pm 177.61$ & 531.64 & 1243.92 & 10.50 & 0.005 \\
\hline & TSCDA & $1130.90 \pm 376.96$ & 668.35 & 1812.25 & & \\
\hline & TSCDI & $1440.80 \pm 699.60$ & 399.31 & 3065.4 & & \\
\hline \multirow[t]{3}{*}{ Chaol index } & TSCDB & $657.89 \pm 107.66$ & 456.21 & 824.95 & 1.768 & 0.413 \\
\hline & TSCDA & $681.25 \pm 120.25$ & 444.71 & 921.43 & & \\
\hline & TSCDI & $637.96 \pm 275.29$ & 229.58 & 1380 & & \\
\hline \multirow[t]{3}{*}{ Simpson's index } & TSCDB & $0.11 \pm 0.07$ & 0.03 & 0.22 & 34.16 & 0.000 \\
\hline & TSCDA & $0.84 \pm 0.22$ & 0.3 & 0.99 & & \\
\hline & TSCDI & $0.54 \pm 0.22$ & 0.22 & 0.95 & & \\
\hline
\end{tabular}

structural destruction of the ileum and colon. These results indicated that the tree shrew could be used for CDI studies.

The gut microbiota of mammals is a complex microbial community that plays an important role in maintaining homeostasis [20]. The indigenous gastrointestinal microbiota protects the host from colonization of pathogenic organisms, and many disease processes are linked to the microbiota [21]. Disruption of the gut microbiota is another factor in $C$. difficile pathogenesis, and several CDI risk factors are associated with the microbiota [22]. In this study, we analyzed the intestinal microbial communnity alterations during the $C$. difficile infection in tree shrews, and compared their features. Recent studies have compared the microbiome profiles of human patients with CDI to healthy controls using next generation sequencing, and have shown a decreased diversity and richness in CDI patients [20, 23]. Generally speaking, CDI patients exhibited lower relative abundances of Lachnospiraceae, Bacteroidiaceae, and Ruminococcaceae with corresponding increases in the relative abundances of Proteobacteria, Lactobacilliaceae, Enterococcaceae, and Streptococcaceae. Perez-Cobas et al. [24] showed that Lactobacillus, Enterococcus, and Clostridium were enriched in CDI patients; this was associated with increases in a group of metabolic processes related to amino acids, proteins, and stress response. Ruminococcaceae, Oscillibacteraceae, Eubacteriaceae and Escherichia were identified as taxa exhibiting potential for $C$. difficile colonization resistance. We found that the response of tree shrew to CDI was similar to that of humans. The relative abundance of many taxa significantly decreased after antibiotic administration and $C$. difficile infection, including Fusobacteria, Bacteroidetes, and Epsilonbacteraeota. The relative abundance of Fusobacteriaceae, Bacteroidaceae, and Helicobacteraceae were lower during infection than during antibiotic treatment; meanwhile the relative abundance of Lachnospiraceae, Enterobacteriaceae, Escherichia, Bacteroides and Clostridium were higher. Ruminococcaceae and Eubacteriaceae showed similar tendencies but were not statistically significant. In addition, the relative abundance of Blautia and Tyzzerella increased after CDI. Consequently, we considered that Lachnospiraceae, Enterobacteriaceae, Bacteroides, Escherichia, Blautia, and Tyzzerella could be used as microbial marker for $C$. difficile infection in tree shrew model. Some of the taxa, such as Enterobacteriaceae and Escherichia were commonly increased both in human and animals after $C$. difficile infection, so these taxa exhibited resistance for $C$. difficile colonization generally. Besides, the metabolic pathways during the infection process of tree shrew also indicated that diversity of the gut microbiota was important for $C$. difficile infection. Before antibiotic treatment and spores gavage, large diversity was found in gastrointestinal tract of tree shrew, and the functional prediction revealed the highest abundance in TSCDB group. After antibiotics usage, the diversity was decreased in TSCDA group, and the abundance of all the COG and KEGG pathways were lowest at this stage, such as energy metabolism, amino acid and carbohydrate metabolism, replication, transcription and defense mechanisms. Thus, the host perhaps was susceptible to pathogen. After $C$. difficile infection, some of the abundance of metabolic pathways 


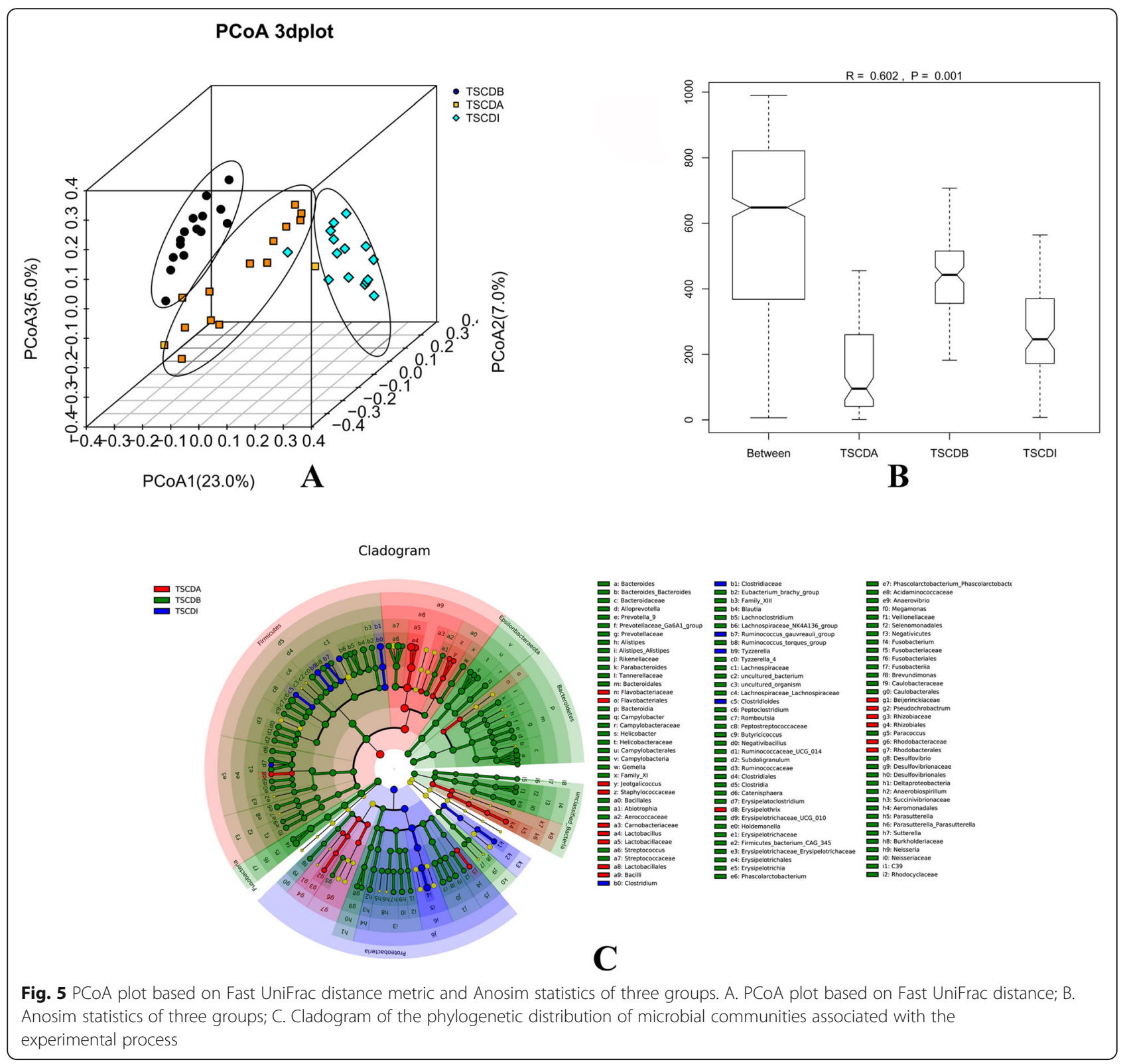

increased, and part of the microbial marker could be identified as mentioned before, and these taxa probably were resistant for $C$. difficile infection.

The increase in relative abundance of Lactobacillus was also interesting. Lactobacillaceae was dominant in CDI samples from both humans and mice [25, 26]. In humans, the presence of Lactobacillus in the intestinal microbiota was generally considered an index of good health and not a trigger for $C$. difficile infection. However, Lactobacillus appeared to be resistant to the antibiotics used in this study. Maybe $C$. difficile infection was favored by a global decrease of alpha diversity, and the overgrowth of Lactobacillus might represent an epiphenomenon due to reduced competition for ecological niche. Lactobacillus remained the dominant taxa after CDI; however, some taxa, such as Escherichia, Streptococcus, Clostridium, Roseburia, and Tyzzerella, did recover.

Reeves et al. [27] used murine model of CDI to demonstrate the changes in endogenous microbial communities against CDI and found a significant change in the structure of the gut microbial community. Antibiotic administration resulted in an outgrowth of Lactobacillaceae. Animals developing clinical symptoms had gut microbiota that were dominated by Proteobacteria. Our results showed similar trends, that was the similar gut microbiota changes during the CDI between murine and tree shrew model. We further summarized the 
Table 2 Differential taxa abundance between TSCDA and TSCDI group in this study

\begin{tabular}{|c|c|c|c|}
\hline & Taxa & $\begin{array}{l}\text { Abundance after } \\
\text { C. difficile infection }\end{array}$ & $P$ value \\
\hline \multirow[t]{4}{*}{ Phylum } & Proteobacteria & Increase & 0.002 \\
\hline & Fusobacteria & Decrease & 0.001 \\
\hline & Bacteroidetes & Decrease & 0.004 \\
\hline & Epsilonbacteraeota & Decrease & 0.002 \\
\hline \multirow[t]{5}{*}{ Class } & Clostridia & Increase & 0.001 \\
\hline & Gammaproteobacteria & Increase & 0.000 \\
\hline & Fusobacteriia & Decrease & 0.001 \\
\hline & Bacteroidia & Decrease & 0.002 \\
\hline & Campylobacteria & Decrease & 0.002 \\
\hline \multirow[t]{5}{*}{ Order } & Clostridiales & Increase & 0.001 \\
\hline & Enterobacteriales & Increase & 0.001 \\
\hline & Fusobacteriales & Decrease & 0.001 \\
\hline & Bacteroidales & Decrease & 0.003 \\
\hline & Campylobacterales & Decrease & 0.002 \\
\hline \multirow[t]{6}{*}{ Family } & Lachnospiraceae & Increase & 0.019 \\
\hline & Enterobacteriaceae & Increase & 0.001 \\
\hline & Clostridiaceae & Increase & 0.000 \\
\hline & Fusobacteriaceae & Decrease & 0.001 \\
\hline & Bacteroidaceae & Decrease & 0.004 \\
\hline & Helicobacteraceae & Decrease & 0.003 \\
\hline \multirow[t]{7}{*}{ Genus } & Escherichia & Increase & 0.001 \\
\hline & Clostridium & Increase & 8.77E-05 \\
\hline & Fusobacterium & Decrease & 0.001 \\
\hline & Bacteroides & Increase & 0.004 \\
\hline & Blautia & Increase & 0.028 \\
\hline & Tyzzerella & Increase & 0.005 \\
\hline & Helicobacter & Decrease & 0.003 \\
\hline
\end{tabular}

characteristics of $C$. difficile infections among tree shrew, rodent animals and human patients (Table 3 ). In this study, the infection method and antibiotics administration were based on previous study, which used the rodents as the animal model. The typical symptoms or signs of diseases were similar between tree shrew and rodents, such as diarrhea or weight loss; however, there was no death of tree shrew. For lesion site of infection, both tree shrew and rodents showed some differences with human patients, as Table 3 shown. In addition, the gut microbiota changes during the infection process were similar among them; the microbial communities of tree shrew, rodents and human were similar before infection, during the antibiotics treatments and even after the infection. Some microbial taxa could be used as commonly $C$. difficile colonization biomarkers, such as Proteobacteria, Enterobacteriaceae and Escherichia.
Therefore, the tree shrew appeared to be a useful model of for CDI, and further studies were warranted.

\section{Conclusions}

The tree shrew is a novel used laboratory animal. Use of the tree shrew as a laboratory animal has increased in recent years due to its low cost of maintenance, life span, small body size, short reproductive cycle, and close relationship to primates. We infected tree shrew with pathogenic $C$. difficile strains. The disease manifestations, histopathology of infections were similar with human and other animal models. The gut microbiota changes during the infection process reflected the important role of gut microbial community in this animal, and mimicked those in humans. All these pieces of evidences indicated tree shrew can be used as a novel animal model for $C$. difficile infection study.

\section{Methods}

Animal sources

Twenty tree shrews were used for this study, which was conducted at the Center of Tree Shrew Germplasm Resources, Institute of Medical Biology, Chinese Academy of Medical Science and Peking Union Medical College in Kunming, China. The tree shrews were from a closed population, and were healthy animals without visible signs of disease or tumors. Ten of the animals were male and 10 were female. The average age was $13.0 \pm 1.5$ months, and ranged from 11 to 15 months. We divided these animals into four groups that were infected with the CD21062, CD10010, or CD12038 strain of C. difficile and one control group without any administration. Each group contained five animals. All of the tree shrews used in this study were the first filial generation and weighed $146.49 \pm 14.60 \mathrm{~g}$. Fecal samples were collected at baseline, during antibiotic treatment, and after $C$. difficile infection. Briefly, fecal samples were collected before antibiotics usage, defined as TSCDB group; feces were collected after antibiotics feeding at day 6, defined as TSCDA group; day 5 of $C$. difficile infection in tree shrew was define as TSCDI group. Total genomic DNA was extracted using a fecal DNA extraction kit (Tiangen, Beijing) according to the manufacturer's instructions [28]. DNA samples were stored at $-20^{\circ} \mathrm{C}$ until they were used for $16 \mathrm{~S}$ rRNA gene sequencing and virulent gene (tcdA, tcdB, $c d t A$, and $c d t B$ ) detection [2].

\section{Bacterial source and spore preparation}

The CD21062, CD10010, and CD12038 strains were all isolated from elderly patients in a tertiary hospital in Beijing, China, and have been previously described [8]. All these strains belonged to ST11, CD21062 was RT078, and others were new ribotypes. All three strains tested positive for the toxin genes $t c d A, t c d B, c d t A$, and 


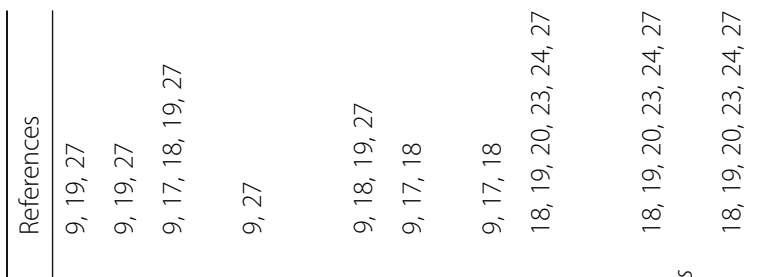
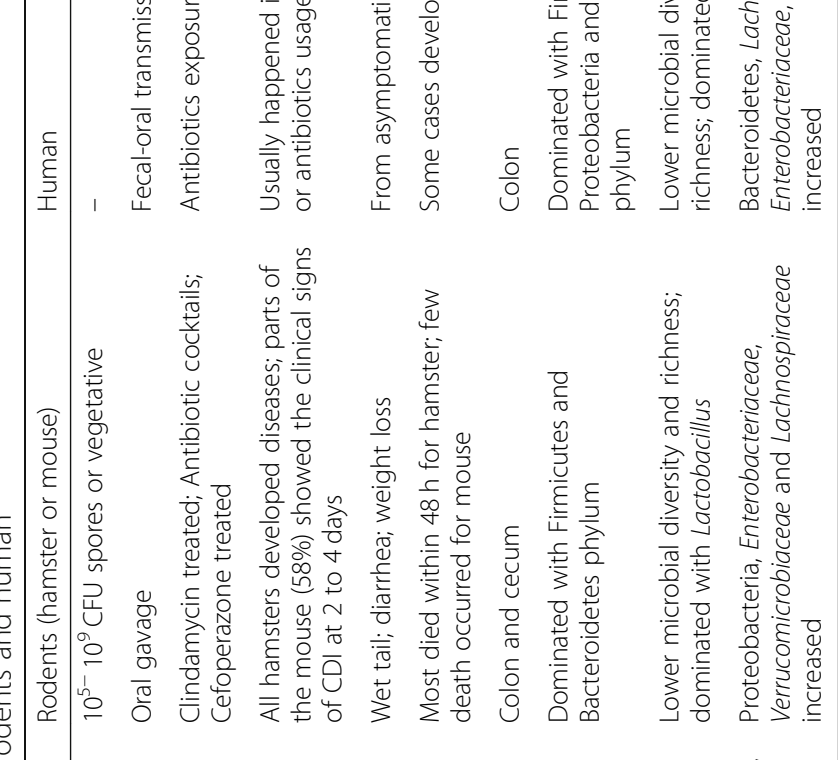

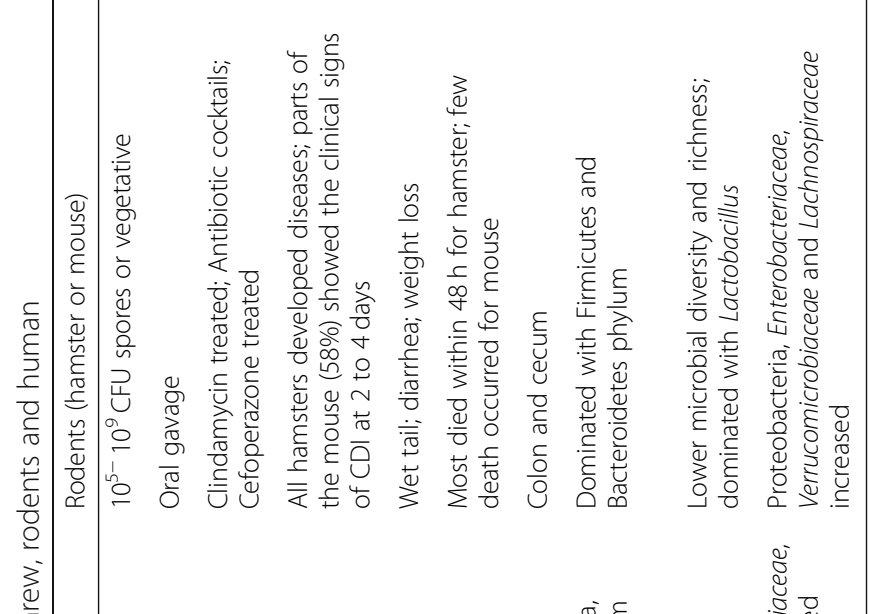

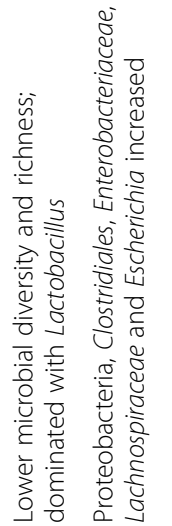


$c d t B$, and the genomes of the strains were deposited in the NCBI database (accession PRJNA497978). The strains were cultured on brain heart infusion (BHI) agar containing 5\% cysteine. An anaerobic environment was maintained at all times using an anaerobic chamber (MITSUBISHI, Japan). An incubation temperature of $37^{\circ} \mathrm{C}$ was used for $\mathrm{C}$. difficile growth.

To prepare spores, strains were incubated under anaerobic conditions for seven days. All surface growth (containing vegetative cells, debris and spores) was extracted and transferred to microcentrifuge tubes containing $1 \mathrm{~mL}$ of sterile ice-cold water. The mixtures were centrifuged five times for $1 \mathrm{~min}$ at $13,000 \times \mathrm{g}$. The washed pellets were suspended in $833 \mu \mathrm{L}$ of $20 \%$ HistoDenz (Sigma-Aldrich) and combined into a $5 \mathrm{~mL}$ mixture. The suspension was gently layered onto $10 \mathrm{~mL}$ of $50 \%$ HistoDenz in a $15 \mathrm{~mL}$ centrifuge tube and centrifuged for 15 $\min$ at $15,000 \times \mathrm{g}$ at $4{ }^{\circ} \mathrm{C}$. The spores were resuspended in $1 \mathrm{~mL}$ of sterile ice-cold water, and centrifuged for $1 \mathrm{~min}$ at $13,000 \times \mathrm{g}$. The procedure was repeated five times to remove HistoDenz, and final pellet was resuspended in $200 \mu \mathrm{L}$ of water [29].

\section{Antibiotic treatment and infection with $C$. difficile}

Tree shrews were housed in 20 sterile cages containing irradiated food and autoclaved water. The experimental animals were received an antibiotic pretreatment, which has been previously described [30]. In general, tree shrews received the antibiotic cocktail for 7 days in the drinking water [metronidazole $(0.215 \mathrm{mg} / \mathrm{mL})$, vancomycin $(0.045 \mathrm{mg} / \mathrm{mL})$, kanamycin $(0.4 \mathrm{mg} / \mathrm{mL})$, gentamicin $(0.035 \mathrm{mg} / \mathrm{mL})$ and colistin $(850 \mathrm{U} / \mathrm{mL})]$. A single intraperitoneal injection of clindamycin $(10 \mathrm{mg} / \mathrm{kg})$ was administered one day prior to infection. Tree shrews were then infected by oral gavage of $10^{5}$ spores of $C$. difficile. The animals were observed daily for signs of disease (i.e., diarrhea or ruffled fur). The tree shrews were euthanized seven days after gavage and the gross anatomy of the abdominal cavities were compared between infected and control ones. The animal intestinal tissues were collected following anesthesia and euthanasia method: tree shrews were cervical dislocated $5 \mathrm{~min}$ following an intraperitoneal injection of $2 \%$ pentobarbital sodium $(0.2 \mathrm{ml} / 100 \mathrm{~g}$, Sigma-Aldrich, USA). Ileal and colonic tissues were collected, washed with PBS, and cut in five pieces for further analyses.

\section{Elisa}

TcdA and TcdB toxins of the infected tree shrew stools were detected by ELISA method. The fresh fecal samples were collected after $C$. difficile infection for one week of all animals. Feces were suspended to $0.5 \mathrm{~g} / \mathrm{ml}$ concentration by using PBS buffer ( $\mathrm{pH}$ 7.4). The fecal suspensions were coated in 96-well plate at $4{ }^{\circ} \mathrm{C}$ overnight, and then washed with PBST (PBS pH 7.4 +0.05\% tween-20). The plate was blocked with $5 \%$ skim milk at $37^{\circ} \mathrm{C}$ for $2 \mathrm{~h}$. The polyclonal antibodies of TcdA and TcdB (List Biological Laboratories, Chicken IgY, 1: 1000 dilution) were used for first hybridization; then Goat anti-Chicken IgY Secondary Antibody, HRP (Invitrogen, 1: 5000 dilution) was used. ELISA-TMB Chromogenic Reagent kit (Sangon, China) was used to determine the absorbance at $450 \mathrm{~nm}$. Pure toxin A and toxin B (List Biological Laboratories) were used as positive controls, and the standard curves were determined at $20 \mathrm{ng} / \mathrm{ml}, 2 \mathrm{ng} / \mathrm{ml}, 0.2 \mathrm{ng} / \mathrm{ml}, 0.02 \mathrm{ng} /$ $\mathrm{ml}$ and $0.002 \mathrm{ng} / \mathrm{ml}$ both for TcdA and TcdB.

\section{Histopathology}

Histopathology was performed to evaluate inflammation and mucosal damage. Resected ileal and colonic tissues were fixed in $4 \%$ paraformaldehyde and stained with hematoxylin-eosin staining (HE) for histological analysis [23].

\section{PCR amplification, library construction, and sequencing} The V3 to V4 variable region of the $16 \mathrm{~S}$ rRNA gene was amplified using previously described primers [31]. Library preparation followed guidelines from Illumina. PCR was performed using the KAPA HiFi HotStart ReadyMix kit (Kapa, Biosystems). The amplification procedure has been previously described. PCR products were purified with AMPure XP magnetic beads (Beckman, Coulter) and quantified using a Qubit fluorometer (Invitrogen, Life Technologies). Secondary PCR amplification was performed to add Illumina Nextera barcodes and the products we purified to remove nontarget fragments. Amplicons were normalized, pooled, and sequencing using an Illumina Miseq system (Illumina, San Diego, USA) [28].

\section{Bioinformatics and statistical analysis}

The raw data were trimmed and low quality $(<\mathrm{Q} 25)$ reads were removed. The paired end reads were merged and barcodes were removed using PEAR 0.9.6, cutadapt 1.2.1, and Prinseq 0.20.4 [32, 33]. QIIME 2.0, USEARCH 11.0 , and R 3.2 were used for bioinformatics analysis [34-36]. Sequences were clustered into operational taxonomic units (OTUs) according to $97 \%$ sequence similarity against the Silva 132 database using the UPARSE pipeline. OTUs were named using SILVA taxonomic nomenclature [37]. Principal coordinate analysis (PCoA) was used to visualize the similarities between the three groups. Analysis of similarities (Anosim) was used to compare the differences in microbial communities between groups. Linear discriminant analysis effect size (LEfSe) analysis was used to identify bacteria with statistically significant $(P<0.05)$ differences in abundance between groups. Phylogenetic investigation of communities 
by reconstruction of unobserved states (PICRUSt) was used to predict the functional potential of the communities. Statistical analyses were performed using SPSS (version 16.0, IBM, USA). The Kolmogorov-Smirnov test, Ttest, or Kruskal-Wallis $\mathrm{H}$ test were used as appropriate. A $P$-value $<0.05$ was considered statistical significance. Sequence data were deposited to the NCBI database (SRA accession: PRJNA541587).

\section{Supplementary information}

Supplementary information accompanies this paper at https://doi.org/10. 1186/s12866-020-01943-z.

Additional file 1. The gross anatomy of the abdominal cavities between infected and control tree shrew. A. Normal control; B. infected animal.

Additional file 2. COG and KEGG pathway annotation results based on PICRUSt.

\section{Abbreviations}

CDI: Clostridioides difficile infection; $\mathrm{BHI}$ : brain heart infusion;

OTUs: operational taxonomic units.; PCOA: Principal co-ordinates analysis;

LEfSe: Linear discriminant analysis Effect Size; PICRUSt: Phylogenetic

investigation of communities by reconstruction of unobserved states

\section{Acknowledgements}

Not applicable.

\section{Authors' contributions}

$J \mathrm{~L}, \mathrm{YW}$ and JD designed the study. Experiments and laboratory analyses were performed by WG, WL, WW, DK, WZ, CL and PT. Analyses were performed by $N L$, YH and XS. WG wrote the paper. All authors read and approved the final manuscript.

\section{Funding}

This work was supported by National Sci-Tech key project (2018ZX10733402 and 2017ZX10103010); Yunnan Science and Technology Talent and Platform Program (2017HC019); Yunnan Joint Support for National Program (2015GA009); Yunnan Province Major Science and Technology Project (2017ZF007); National Natural Science Foundation of China (NSFC) (U1702282). The funders have no role in the design, collection, analysis, interpretation of data and in writing the manuscript of the study.

\section{Availability of data and materials}

The datasets generated and/or analysed during the current study are available in the NCBI database repository by the SRA accession: PRJNA541587, [https://www.ncbi.nlm.nih.gov/bioproject/PRJNA541587].

\section{Ethics approval and consent to participate}

The animal experiments and detection protocols were carried out in accordance with relevant guidelines and regulations, and were approved by the Ethics Committee of the Institute of Medical Biology, Chinese Academy of Medical Sciences and Peking Union Medical College. All experimental procedures were approved by the Ethics Review Committee [Institutional Review Board (IRB)] of the Institute of Medical Biology, Chinese Academy of Medical Sciences and Peking Union Medical College.

\section{Consent for publication}

Not applicable.

\section{Competing interests}

The authors declare that they have no competing interests.

\section{Author details}

${ }^{1}$ Center of Tree Shrew Germplasm Resources, Institute of Medical Biology, Chinese Academy of Medical Sciences and Peking Union Medical College, Yunnan Key Laboratory of Vaccine Research and Development on Severe
Infectious Diseases, Yunnan Innovation Team of Standardization and Application Research in Tree Shrew, Zhao zong Road 66, Kunming 650118, China. ${ }^{2}$ Department of Acute Infectious Diseases Control and Prevention, Yunnan Provincial Centre for Disease Control and Prevention, Kunming 650022, China. ${ }^{3}$ State Key Laboratory of Infectious Disease Prevention and Control, Collaborative Innovation Center for Diagnosis and Treatment of Infectious Diseases, National Institute for Communicable Disease Control and Prevention, Chinese Center for Disease Control and Prevention, Chang bai Road 155, Chang ping District, Beijing 102206, China.

Received: 6 April 2020 Accepted: 11 August 2020

Published online: 20 August 2020

References

1. Liu XS, Li WG, Zhang WZ, Wu Y, Lu JX. Molecular characterization of Clostridium difficile isolates in China from 2010 to 2015. Front Microbiol. 2018;9:845.

2. Liao F, Li W, Gu W, Zhang W, Liu X, Fu X, Xu W, Wu Y, Lu J. A retrospective study of community-acquired Clostridium difficile infection in Southwest China. Sci Rep. 2018:8(1):3992.

3. Davies KA, Longshaw CM, Davis GL, Bouza E, Barbut F, Barna Z, Delmee M, Fitzpatrick F, Ivanova K, Kuijper $\mathrm{E}$, et al. Underdiagnosis of Clostridium difficile across Europe: the European, multicentre, prospective, biannual, point-prevalence study of Clostridium difficile infection in hospitalised patients with diarrhoea (EUCLID). Lancet Infect Dis. 2014;14(12):1208-19.

4. Warny M, Pepin J, Fang A, Killgore G, Thompson A, Brazier J, Frost E, McDonald LC. Toxin production by an emerging strain of Clostridium difficile associated with outbreaks of severe disease in North America and Europe. Lancet. 2005;366(9491):1079-84.

5. Knight DR, Elliott B, Chang BJ, Perkins TT, Riley TV. Diversity and evolution in the genome of Clostridium difficile. Clin Microbiol Rev. 2015;28(3):721-41.

6. Lim SK, Stuart RL, Mackin KE, Carter GP, Kotsanas D, Francis MJ, Easton M, Dimovski K, Elliott B, Riley TV, et al. Emergence of a ribotype 244 strain of Clostridium difficile associated with severe disease and related to the epidemic ribotype 027 strain. Clin Infect Dis. 2014;58(12):1723-30.

7. Jin H, Ni K, Wei L, Shen L, Xu H, Kong Q, Ni X. Identification of Clostridium difficile RT078 from patients and environmental surfaces in Zhejiang Province, China. Infect Control Hosp Epidemiol. 2016;37(6):745-6.

8. Suo J, Yan Z, Wu Y, Li WG, Zhang WZ, Liu XS, Liu Y, Lu J. Clostridium difficile RT 078/ST11: a threat to community population and pigs identified in elder hospitalized patients in Beijing, China. Infect Control Hosp Epidemiol. 2017; 38(11):1383-5

9. Best EL, Freeman J, Wilcox MH. Models for the study of Clostridium difficile infection. Gut Microbes. 2012;3(2):145-67.

10. Hutton ML, Mackin KE, Chakravorty A, Lyras D. Small animal models for the study of Clostridium difficile disease pathogenesis. FEMS Microbiol Lett. 2014;352(2):140-9

11. Gu W, Tong P, Liu C, Wang W, Lu C, Han Y, Sun X, Kuang X, Li N, Dai J. The characteristics of gut microbiota and commensal Enterobacteriaceae isolates in tree shrew (Tupaia belangeri). BMC Microbiol. 2019;19(1):203.

12. Fan Y, Huang ZY, Cao CC, Chen CS, Chen YX, Fan DD, He J, Hou HL, Hu L, Hu XT, et al. Genome of the Chinese tree shrew. Nat Commun. 2013:4:1426.

13. Yao YG. Creating animal models, why not use the Chinese tree shrew (Tupaia belangeri chinensis)? Zool Res. 2017;38(3):118-26.

14. Pickard JM, Zeng MY, Caruso R, Nunez G. Gut microbiota: role in pathogen colonization, immune responses, and inflammatory disease. Immunol Rev. 2017;279(1):70-89.

15. Bartlett JG, Onderdonk AB, Cisneros RL, Kasper DL. Clindamycin-associated colitis due to a toxin-producing species of Clostridium in hamsters. J Infect Dis. 1977;136(5):701-5.

16. Lawley TD, Clare S, Walker AW, Goulding D, Stabler RA, Croucher N, Mastroeni P, Scott P, Raisen C, Mottram L, et al. Antibiotic treatment of clostridium difficile carrier mice triggers a supershedder state, sporemediated transmission, and severe disease in immunocompromised hosts. Infect Immun. 2009;77(9):3661-9.

17. Buffie CG, Jarchum I, Equinda M, Lipuma L, Gobourne A, Viale A, Ubeda C, Xavier J, Pamer EG. Profound alterations of intestinal microbiota following a single dose of clindamycin results in sustained susceptibility to Clostridium difficile-induced colitis. Infect Immun. 2012;80(1):62-73.

18. Theriot CM, Koenigsknecht MJ, Carlson PE Jr, Hatton GE, Nelson AM, Li B, Huffnagle GB. J ZL, Young VB: antibiotic-induced shifts in the mouse gut 
microbiome and metabolome increase susceptibility to Clostridium difficile infection. Nat Commun. 2014;5:3114.

19. Theriot CM, Koumpouras CC, Carlson PE, Bergin II, Aronoff DM, Young VB. Cefoperazone-treated mice as an experimental platform to assess differential virulence of Clostridium difficile strains. Gut Microbes. 2011;2(6): 326-34.

20. Engevik MA, Yacyshyn MB, Engevik KA, Wang J, Darien B, Hassett DJ, Yacyshyn BR, Worrell RT. Human Clostridium difficile infection: altered mucus production and composition. Am J Physiol Gastrointest Liver Physiol. 2015;308(6):G510-24.

21. Kamada N, Chen GY, Inohara N, Nunez G. Control of pathogens and pathobionts by the gut microbiota. Nat Immunol. 2013;14(7):685-90.

22. Ross CL, Spinler JK, Savidge TC. Structural and functional changes within the gut microbiota and susceptibility to Clostridium difficile infection. Anaerobe. 2016:41:37-43.

23. Milani C, Ticinesi A, Gerritsen J, Nouvenne A, Lugli GA, Mancabelli L, Turroni F, Duranti S, Mangifesta M, Viappiani A, et al. Gut microbiota composition and Clostridium difficile infection in hospitalized elderly individuals: a metagenomic study. Sci Rep. 2016;6:25945.

24. Perez-Cobas AE, Artacho A, Ott SJ, Moya A, Gosalbes MJ, Latorre A. Structural and functional changes in the gut microbiota associated to Clostridium difficile infection. Front Microbiol. 2014:5:335.

25. Rea MC, Dobson A, O'Sullivan O, Crispie F, Fouhy F, Cotter PD, Shanahan F, Kiely B, Hill C, Ross RP. Effect of broad- and narrow-spectrum antimicrobials on Clostridium difficile and microbial diversity in a model of the distal colon. Proc Natl Acad Sci U S A. 2011:108(Suppl 1):4639-44.

26. Antharam VC, Li EC, Ishmael A, Sharma A, Mai V, Rand KH, Wang GP. Intestinal dysbiosis and depletion of butyrogenic bacteria in Clostridium difficile infection and nosocomial diarrhea. J Clin Microbiol. 2013;51(9):288492.

27. Reeves AE, Theriot CM, Bergin IL, Huffnagle GB, Schloss PD, Young VB. The interplay between microbiome dynamics and pathogen dynamics in a murine model of Clostridium difficile infection. Gut Microbes. 2011;2(3):14558.

28. Liao F, Gu W, Li D, Liang J, Fu X, Xu W, Duan R, Wang X, Jing H, Dai J: Characteristics of microbial communities and intestinal pathogenic bacteria for migrated Larus ridibundus in southwest China. Microbiologyopen 2018: e00693.

29. Sorg JA, Dineen SS: Laboratory maintenance of Clostridium difficile. Curr Protoc Microbiol 2009, Chapter 9:Unit9A 1.

30. Chen X, Katchar K, Goldsmith JD, Nanthakumar N, Cheknis A, Gerding DN, Kelly CP. A mouse model of Clostridium difficile-associated disease. Gastroenterology. 2008;135(6):1984-92.

31. Klindworth A, Pruesse E, Schweer T, Peplies J, Quast C, Horn M, Glockner FO. Evaluation of general $16 \mathrm{~S}$ ribosomal RNA gene PCR primers for classical and next-generation sequencing-based diversity studies. Nucleic Acids Res. 2013; 41(1):e1.

32. Schmieder R, Edwards R. Quality control and preprocessing of metagenomic datasets. Bioinformatics. 2011;27(6):863-4.

33. Zhang J, Kobert K, Flouri T, Stamatakis A. PEAR: a fast and accurate Illumina paired-end reAd mergeR. Bioinformatics. 2014;30(5):614-20.

34. Caporaso JG, Kuczynski J, Stombaugh J, Bittinger K, Bushman FD, Costello EK, Fierer N, Pena AG, Goodrich JK, Gordon Jl, et al. QIIME allows analysis of high-throughput community sequencing data. Nat Methods. 2010;7(5):3356.

35. Edgar RC. UPARSE: highly accurate OTU sequences from microbial amplicon reads. Nat Methods. 2013;10(10):996-8.

36. Cao Y, Zheng X, Li F. Bo X: mmnet: an R package for Metagenomics systems biology analysis. Biomed Res Int. 2015;2015:167249.

37. Quast C, Pruesse E, Yilmaz P, Gerken J, Schweer T, Yarza P, Peplies J, Glockner FO. The SILVA ribosomal RNA gene database project: improved data processing and web-based tools. Nucleic Acids Res. 2013;41(Database issue):D590-6.

\section{Publisher's Note}

Springer Nature remains neutral with regard to jurisdictional claims in published maps and institutional affiliations.

\section{Ready to submit your research? Choose BMC and benefit from}

- fast, convenient online submission

- thorough peer review by experienced researchers in your field

- rapid publication on acceptance

- support for research data, including large and complex data types

- gold Open Access which fosters wider collaboration and increased citations

- maximum visibility for your research: over $100 \mathrm{M}$ website views per year

At $\mathrm{BMC}$, research is always in progress.

Learn more biomedcentral.com/submissions 